\title{
Tratamiento complementario de heridas crónicas con factor de crecimiento estimulante de colonias de granulocitos. A propósito de dos casos clínicos.
}

\author{
Granulocyte Colony-Stimulating Growth Factor complementary treatment \\ in chronic wounds. Report of two cases. \\ Tratamento complementar de feridas crônicas com colônias de \\ granulócitos estimulantes do fator de crescimento. Cerca de dois casos \\ clínicos.
}

Natalia Ubillos Dermatóloga Médico- Quirúrgica.

Begoña Valdés

Dermatóloga Médico- Quirúrgica

Lourdes Echarte

Magister en Ciencias Biológicas.

Asistente del Área de Terapia

Celular y Medicina Regenerativa.

Departamento Básico de Medicina

Alexandra Sujanov

Especialista en Medicina

Transfusional. Asistente del Área

de Terapia Celular y Medicina

Regenerativa.

CarolineAgorio

Dermatóloga. Profesora Adjunta de la Cátedra de Dermatología MédicoQuirúrgica.

Cristina Touriño

Hematóloga. Profesora Agregada del Departamento Básico de Medicina. Coordinadora del Área de Terapia

Celular y Medicina Regenerativa.

Lilian Díaz

Hematóloga. Profesora titular de Cátedra de Hematología.

Gabriela Otero

Dermatóloga. Ex Asistente de la Cátedra de Dermatología MédicoQuirúrgica.
Resumen: Introducción. Independientemente de su etiología, las heridas crónicas, representan un desafío terapéutico debido a que muchas veces son refractarias a tratamientos convencionales. Es por este motivo que en los últimos años se han desarrollado de forma creciente estrategias complementariaseneláreadelamedicinaregenerativa, como:terapiasconcélulasmadre, ingeniería de tejidos, plasma rico en plaquetas y factores de crecimiento aplicados en las heridas crónicas.

Dichas terapias complementarias han mostrado ciertos beneficios en la cicatrización de heridas complejas. Materiales y métodos. Se presentan dos casos clínicos de pacientes con heridas crónicas de diferente etiología, refractarias al tratamiento con cura avanzada de heridas, las cuales recibieron de forma complementaria factor estimulante de colonias de granulocitos (G-CSF; Filgen $($ ). Se realizaron inyecciones locales de G-CSF a una dosis de $300 \mathrm{mcg} / \mathrm{ml}$ en piel peri úlcera en forma semanal completando dos series de cuatro inyecciones cada una. Resultados. Ambos casos presentaron una reducción en el área de las mismas alcanzándose la cicatrización total en un paciente y una reducción del $37 \%$ en el otro luego de dos series. El procedimiento fue bien tolerado y no se reportaron efectos adversos relacionados al mismo. Conclusiones. Los resultados obtenidos en estos pacientes mostraron beneficios en la cicatrización con la aplicación del G-CSF. Se requieren de ensayos clínicos controlados que permitan establecer el rol de dicho factor en el tratamiento de las mismas. Por este motivo nuestro grupo de trabajo se encuentra desarrollando un protocolo que permita evaluar este aspecto.

Palabras clave: heridas crónicas, factor de crecimiento estimulante de colonias de granulocitos, G-CSF, cicatrización, medicina regenerativa.

Abstract: Introduction. Regardless of their etiology, chronic wounds, represent a therapeutic challenge because they are often refractory to conventional treatments. Due to this observation, in the last few years, new complementary strategies have emerged in the area of regenerative medicine, including stem cell therapeutics, tissue engineering, platelet rich plasma, and growth factors applied to chronic wounds. These complementary therapies have shown certain benefits in healing of complex wounds. Materials and methods. We present two clinical cases of patients with chronic wounds of different etiology, refractory to advanced and conventional wound treatments, which received complementary granulocyte colony-stimulating growth factor (G-CSF; Filgen $®)$. Local injections with G-CSF were administered weekly in periulcer skin at a dose of $300 \mathrm{mcg} / \mathrm{ml}$, completing two series of four injections each. Results. Both cases showeda reduction in their areas, reaching to total healing inone patient, and a reduction of $37 \%$ in the other one after two series of treatment. The procedure was well tolerated and no adverse effects were detected. Conclusions. The results obtained with these patients showed a benefit in cicatrization with the administration of G-CSF. Controlled clinical trials are needed to establish the role of G-CSF in these wounds. Thus, our group is developing a protocol to evaluate this aspect. 
Keywords: chronic wounds, granulocyte colony-stimulating growth factor, G-CSF, cicatrization, wound healing, regenerative medicine.

Resumo: Introdução. Independentemente da sua etiologia, as feridas crônicas representam um desafio terapêutico porque muitas vezes são refratárias aos tratamentos convencionais. É por esta razão que nos últimos anos foram desenvolvidas estratégias complementares na área da medicina regenerativa, tais como: terapias de células estaminais, engenharia de tecidos, plasma rico em plaquetas e fatores de crescimento aplicados a feridas crônicas. Tais terapias complementares mostraram certos benefícios na cicatrização de feridas complexas. Materiais e métodos. Dois casos de pacientes com feridas crónicas de diferentes etiologias, refractárias ao tratamento com a cura da ferida avançado, que receberam complementaria factor estimulante forma de colónias de granulócitos (Filgen ${ }^{\circledR}$ FEC-G) presente. As injeções locais de G-CSF foram feitas a uma dose de $300 \mathrm{mcg} / \mathrm{ml}$ na pele peri úlcera, semanalmente, completando duas séries de quatro injeções cada. Resultados. Ambos os casos mostraram uma redução na área do mesmo atingindo a cicatrização total em um paciente e uma redução de $37 \%$ no outro após duas séries. O procedimento foi bem tolerado e nenhum efeito adverso relacionado a ele foi relatado. Conclusões. Os resultados obtidos nestes pacientes mostraram benefícios na cura com a aplicação de G-CSF. Ensaios clínicos controlados são necessários para estabelecer o papel desse fator no tratamento deles. Por esse motivo, nosso grupo de trabalho está desenvolvendo um protocolo que nos permite avaliar esse aspecto.

Palavras-chave: feridas crônicas, colônias de granulócitos estimulantes do fator de crescimento, G-CSF, cicatrização, medicina regenerativa.

Recibido: 13/06/2017 - Aceptado: 19/02/2018

Cátedra de Dermatología Médico Quirúrgica. Hospital de Clínicas "Dr. Manuel Quintela" Facultad de Medicina. Universidad de la República. Montevideo- Uruguay.

Correspondencia: E- mail: nubi212@gmail.com, nataliaubillos@gmail.com 


\section{Introducción}

Las úlceras crónicas son un problema de salud, con importante morbilidad, alteración en la calidad de vida, aumento del ausentismo laboral así como un gran impacto económico sobre el sistema de salud. ${ }^{(1-3)}$

Se estima que esta patología afecta a 6,5 millones de pacientes en Estados Unidos con un costo anual de 7 billones de dólares para el sistema de salud de dicho país mientras que en Europa los gastos anuales oscilan entre 25 a 200 millones de dólares. ${ }^{(4,5)}$

En Uruguay no contamos con datos epidemiológicos sobre la magnitud del problema, si bien los trabajos de Ramos y cols y posteriormente Otero y cols aportan datos sobre su etiología en unidades de referencia de Montevideo. ${ }^{(2,3)}$

La cicatrización de heridas es un proceso dinámico que consta de tres fases superpuestas en el tiempo, ellas son: fase inflamatoria, proliferativa y de remodelación. Éstas involucran la interacción dinámica entre factores de crecimiento, citoquinas, componentes de la matriz extracelular, células residentes y subtipos diferentes de linfocitos. ${ }^{(6)}$

La alteración en alguna de estas fases, generalmente de causa multifactorial, puede conducir a la cronicidad de las heridas. ${ }^{(1)}$

Varios ensayos clínicos han demostrado que en los fluidos de úlceras crónicas los factores de crecimiento se encuentran disminuidos en comparación con las heridas agudas. ${ }^{(7)}$ Esto motivó diversos estudios prospectivos, utilizando factores de crecimiento aplicados en forma local a pacientes con heridas crónicas, obteniendo resultados alentadores con respecto a la cicatrización. ${ }^{(7-9)}$ Los resultados de los mismos se muestran en la tabla 1.

\begin{tabular}{|l|l|l|l|l|l|}
\hline Autores & Estudio & $\begin{array}{l}\text { Via de } \\
\text { administración }\end{array}$ & $\begin{array}{l}\text { Dosis } \\
\text { Ng }\end{array}$ & Series & Resultado \\
\hline $\begin{array}{l}\text { Marques da Costa } \\
\text { et al. }\end{array}$ & $\begin{array}{l}\text { Randomizado doble } \\
\text { ciego con placebo } \\
\text { (úlceras venosas y } \\
\text { otras etiologías) }\end{array}$ & Subcutánea & 400 & Única & $\begin{array}{l}\text { Diferencias en } \\
\text { tamaño, cicatrización } \\
\text { y síntomas entre los } \\
\text { grupos }\end{array}$ \\
\hline Jaschke et al. & Intervención & Tópica & $\begin{array}{l}5 \text { en } \\
\text { NaCl }\end{array}$ & $\begin{array}{l}\text { 2-3 veces } \\
\text { semana }\end{array}$ & $\begin{array}{l}\text { Tasas de curación del } \\
90 \text { en un promedio } \\
\text { de } 19 \text { semanas }\end{array}$ \\
\hline Cianfarani et al. & $\begin{array}{l}\text { Intervención: } \\
\text { Biopsia de úlcera }\end{array}$ & Intradérmica & 150 & Semanal & $\begin{array}{l}\text { Aumento en la angio- } \\
\text { génesis y del tejido de } \\
\text { granulación }\end{array}$ \\
\hline
\end{tabular}

$\mathrm{NaCl}$ : Cloruro de sodio

Dentro de los factores de crecimiento evaluados en éstas heridas se encuentra el factor de crecimiento estimulante de colonias de granulocitos y macrófagos (GM-CSF). Éste es una citoquina multifuncional que actúa regulando la proliferación y diferenciación de células madre y progenitoras hematopoyéticas y endoteliales desde la médula ósea, así como células no hematopoyéticas como fibroblastos, células endoteliales y queratinocitos. ${ }^{(7-9)}$

Cianfarani y cols. evaluaron los efectos del GM-CSF sobre la neo-angiogénesis de úlceras venosas crónicas y la síntesis local de factores pro-angiogénicos. Con este fin, aplicaron GMCSF intradérmico en cuatro puntos cardinales de la lesión, a una dosis de 150 microgramos $(\mu \mathrm{g})$, en forma semanal, con un total de cuatro a nueve dosis. Como conclusión destacan el aumento de la angiogénesis y del tejido de granulación en la herida, vinculado a un aumento local de factores angiogénicos, como el factor de crecimiento endotelial vascular (VEGF). ${ }^{(9)}$

Posteriormente, diversos autores comenzaron a utilizar factor de crecimiento estimulante de colonias de granulocitos (G-CSF), primero en modelo animal y luego en pacientes con pie diabético infectado. ${ }^{(10-15)}$

El G-CSF, al igual que el GM-CSF, es un factor endógeno hematopoyético movilizador de células madre y progenitores hematopoyéticos de la médula ósea hacia la sangre periférica, los cuales pueden ser cuantificados mediante la determinación de células CD34+ en sangre periférica. ${ }^{(10,12)}$

A nivel sistémico actúa regulando la supervivencia, proliferación y diferenciación terminal de las células precursoras de neutrófilos desde la médula a la sangre. ${ }^{(16)}$ 
A su vez, estimula la función de neutrófilos maduros favoreciendo la quimiotaxis de éstos y mejorando su capacidad fagocítica. ${ }^{(10,12,17)}$

Por otro lado, induce la proliferación de células endoteliales promoviendo la angiogénesis. ${ }^{(10)}$

La eficacia del G-CSF ha sido evaluada en varios ensayos clínicos en pie diabético infectado, observándose mejoría en los parámetros de infección, así como disminución en la incidencia de amputación y de estadía hospitalaria en estos pacientes. ${ }^{(12-14)}$

En contra posición a esto, Yönet y cols. no lograron demostrar efectos beneficiosos de significancia con la administración subcutánea de G-CSF, aunque si evidenciaron aumento en el número absoluto de neutrófilos y la eficacia en su función. ${ }^{(15)}$

La dosis utilizada, así como el intervalo de aplicación fue variable entre los distintos trabajos, oscilando entre 5 a $10 \mu \mathrm{g} / \mathrm{kg}$ de G-CSF aplicados en forma diaria durante un periodo de tres a veintiún días. ${ }^{(12-15)}$

Se reportó buena tolerancia con escasos efectos adversos, aunque Kästenbauer y cols. reportaron alteraciones hepáticas en dos pacientes del grupo tratado con G-CSF por lo que se debió suspender su administración. ${ }^{(13)}$

En una revisión de Cochrane, Cruciane y cols. Evaluaron los efectos in vivo sobre las tasas de infección y cicatrización del tratamiento complementario con G-CSF comparado con placebo en pacientes con pie diabético infectado. Los autores sugieren que la aplicación de G-CSF no mejoraría la cicatrización ni aumentaría la probabilidad de resolución de infecciones. Sin embargo, refieren una disminución en la necesidad de amputación y en el tiempo de internación. Concluyen por tanto que éste tratamiento podría ser incorporado a las pautas de tratamiento habitual del pie diabético infectado, sobre todo en aquellas infecciones que pongan en riesgo la viabilidad de la extremidad. ${ }^{(17)}$

Por otra parte, en úlceras de otras etiologías, Sugiyama y cols. realizaron un estudio en modelo animal de heridas producidas por radiación. Para ello utilizaron una combinación de factores de crecimiento entre los cuales se encuentra el G-CSF, factor estimulante de colonias de macrófagos (M-GCS) y un inhibidor del factor de crecimiento transformante $\beta-1$ (TGF- $\beta 1$ ). Estos autores observaronun aumento en la resistencia a la ruptura de la piel y de la formación de fibras de colágeno en la histopatología y concluyeron que la combinación de factores podría ser prometedor para el tratamiento de heridas producidas por radiación. ${ }^{(18)}$

Fine y cols. en un ensayo clínico piloto con siete pacientes utilizaron G-CSF en forma sub cutánea en heridas de epidermólisis ampollar a una dosis de $10 \mu \mathrm{g} / \mathrm{kg} /$ díapor siete días. Observaron una reducción del 75,5\% en el tamaño de las lesiones y del 36,6 \% en el recuento de erosiones y ampollas al finalizar la serie. ${ }^{(19)}$

Basándonos en la evidencia presentada, se planteó la utilización de G-CSF (Filgen ${ }^{\circledR}$ ) en dos pacientes con heridas crónicas de diferente etiología, las cuales mantenían su cronicidad a pesar de los esfuerzos clínicos para estimular la correcta cicatrización.

Este producto se presenta en jeringas pre llenadas de 300 micro gramos ( $\mu$ grs) por mililitro (ml). (Filgrastim - Filgen ${ }^{\circledR}$ ) La técnica que se utilizó para la aplicación del G-CSF en ambos casos, consistió en la división de la herida en cuatro cuadrantes, inyectando 0,25 ml intradérmicos a 0,5 $\mathrm{cm}$ del borde en cada cuadrante. A su vez, se realizó extracción de sangre para cuantificación de células CD34+ por citometría de flujo, previo a la primera inyección y a la semana, no evidenciándose efectos sistémicos del G-CSF sobre dicho marcador en ninguno de los casos.

Los dos pacientes recibieron un total de cuatro aplicaciones semanales divididas en dos series. El período entre las series fue de dieciocho semanas para el caso uno y cuatro semanas en el caso dos.

El protocolo de utilización del G- CSF como tratamiento complementario de úlceras venosas crónicas fue aprobado por el Comité de Ética del Hospital de Clínicas. Ambos pacientes recibieron información del procedimiento previo al mismo y dieron su consentimiento informado para realizar dicho tratamiento. 


\section{Caso clínico 1}

Hombre de 76 años, con antecedentes de hipertensión, diabetes tipo II, dislipemia, hiperuricemia. Al momento de la consulta, presentaba una úlcera venosa crónica de miembro inferior izquierdo de veinte años de evolución que habiendo recibidomúltiples tratamientos, entre ellos injerto de piel y terapia con oxigeno hiperbárico, sólo se obtuvo una respuesta parcial. Los datos clínicos se detallan en la tabla 2.

\begin{tabular}{|l|l|l|}
\hline Paciente & $\mathbf{1}$ & $\mathbf{2}$ \\
\hline Etiología & Úlcera venosa & Cicatriz quirúrgica \\
\hline Localización & Miembro inferior izquierdo & Parietal izquierdo \\
\hline Series de 4 inyecciones de G-CSF & 2 & 2 \\
\hline Período entre series & 18 semanas & 4 semanas \\
\hline Leucocitos mil/mm3* & $8,82 / 9,24$ & $5,04 / 6,92$ \\
\hline CD34 \% * & $0,03 / 0,02$ & $0,06 / 0,06$ \\
\hline
\end{tabular}

* pre tratamiento/post tratamiento

Debido a la falta de progresión en el proceso cicatrización, se decidió realizar, como técnica complementaria, la aplicación de G-CSF a nivel local (Figura 1). No se evidenció efectos adversos de relevancia durante el procedimiento.
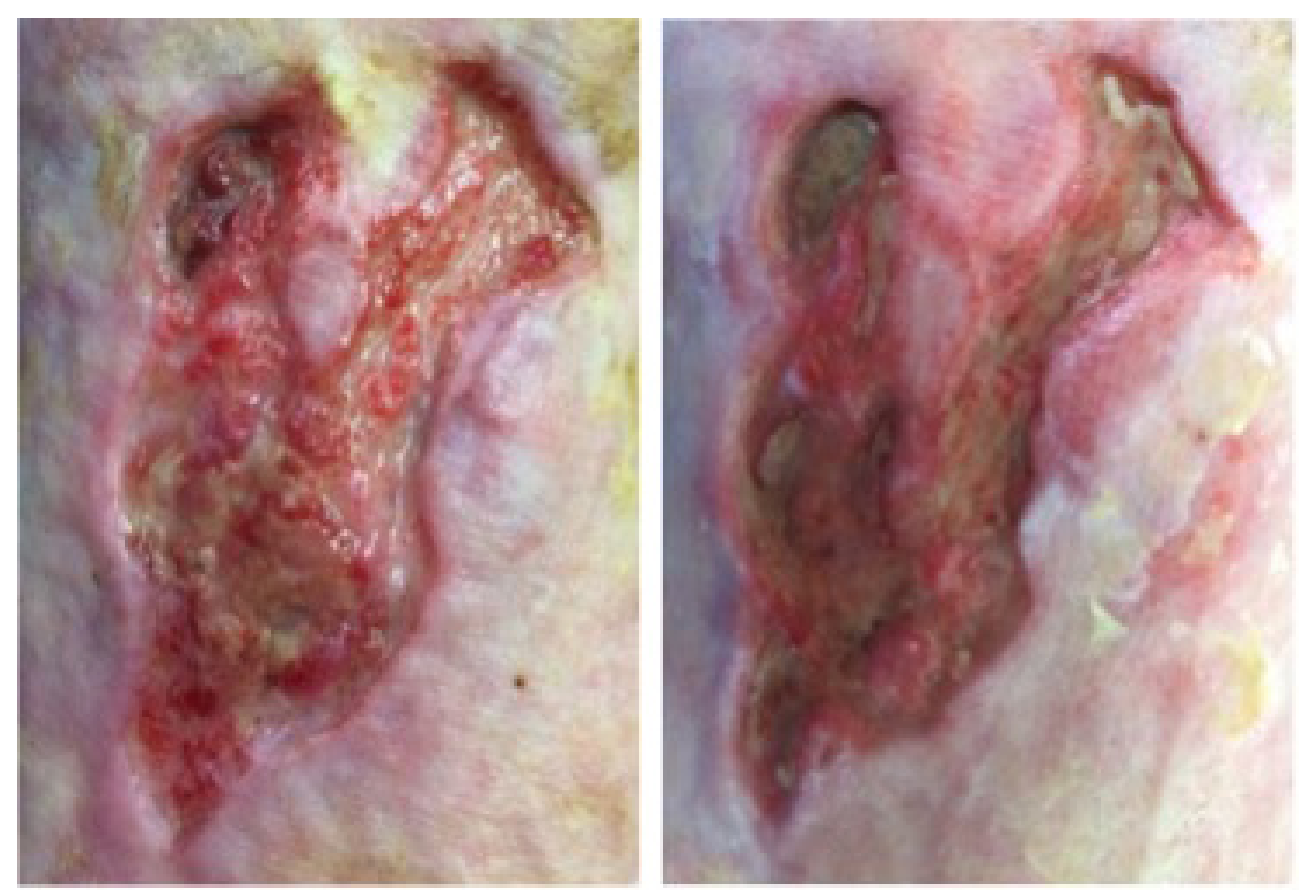

Durante veintiséis semanas de seguimiento se observó una reducción porcentual del área del 37\%. (Tabla 3 y Gráfico 1)

\begin{tabular}{|l|l|l|}
\hline Paciente & $\mathbf{1}$ & $\mathbf{2}$ \\
\hline Área pre tratamiento con G-CSF & $28,8^{*}$ & 1,52 * \\
\hline Área luego de la primera serie & $22,4^{*}$ & $1,13^{*}$ \\
\hline Área previo a segunda serie & 22,3 & $0,4^{*}$ \\
\hline Área luego de segunda serie & 18,1 & 0,02 * \\
\hline Área control al mes & 18,1 & 0 * \\
\hline$\%$ de reducción de área en 6 meses & 37,1 & 100 \\
\hline
\end{tabular}

* Área expresada en centímetros cuadrados (cm2) 


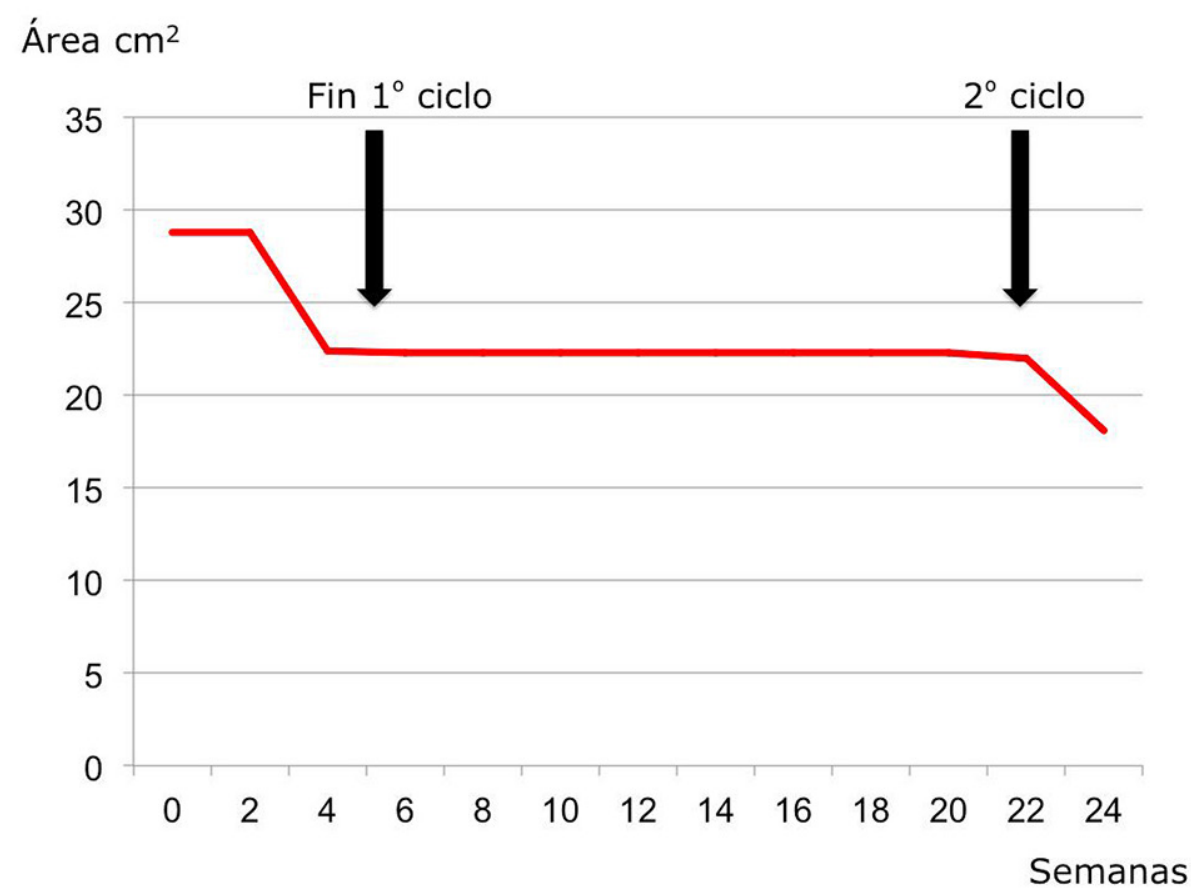

\section{Caso clínico 2}

Hombre de 59 años con antecedente de tumor cerebral en la infancia tratado con cirugía y radioterapia en esa oportunidad. Un año previo a la consulta, presentó un carcinoma basocelular en región parietal izquierda de cuero cabelludo en área previamente irradiada, recibiendo tratamiento quirúrgico de dicha lesión. El estudio anátomo - patológico confirmó el diagnóstico planteado inicialmente, estando los márgenes libres de tumor.

Se realizó reconstrucción del defecto quirúrgico por colgajo de avance, el cual sufrió necrosis parcial, requiriendo, posteriormente, cura avanzada de heridas con apósito hidrocoloide. Durante el seguimiento presentó una tórpida evolución en la cicatrización con exposición de material óseo. Dado que se trataba de un hueso irradiado, lo que dificultaba la posibilidad de un injerto, se planteó la realización de inyección de G-CSF. (Figura 2)

Figura 2: A la izquierda la lesión pre tratamiento con G-CSF. A la derecha lesión luego de ocho sesiones de G-CSF
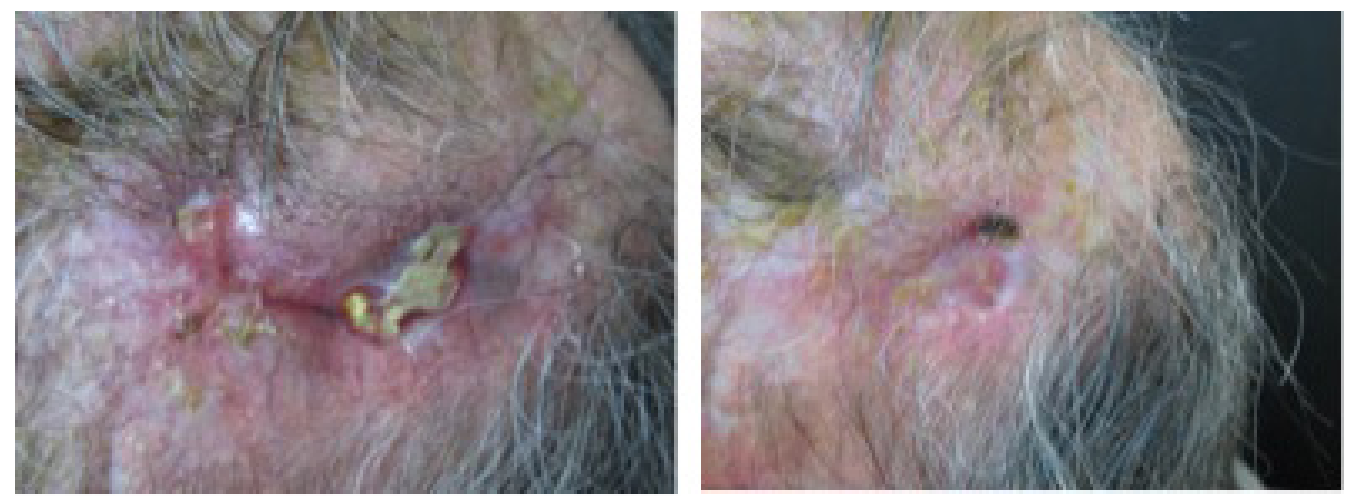

Los datos clínicos y evaluación paraclínica se detallan en la tabla 2.

Durante las veinticuatro semanas de seguimiento se observó una reducción porcentual del área del 100\%. (Tabla 3 y Gráfico 2). 


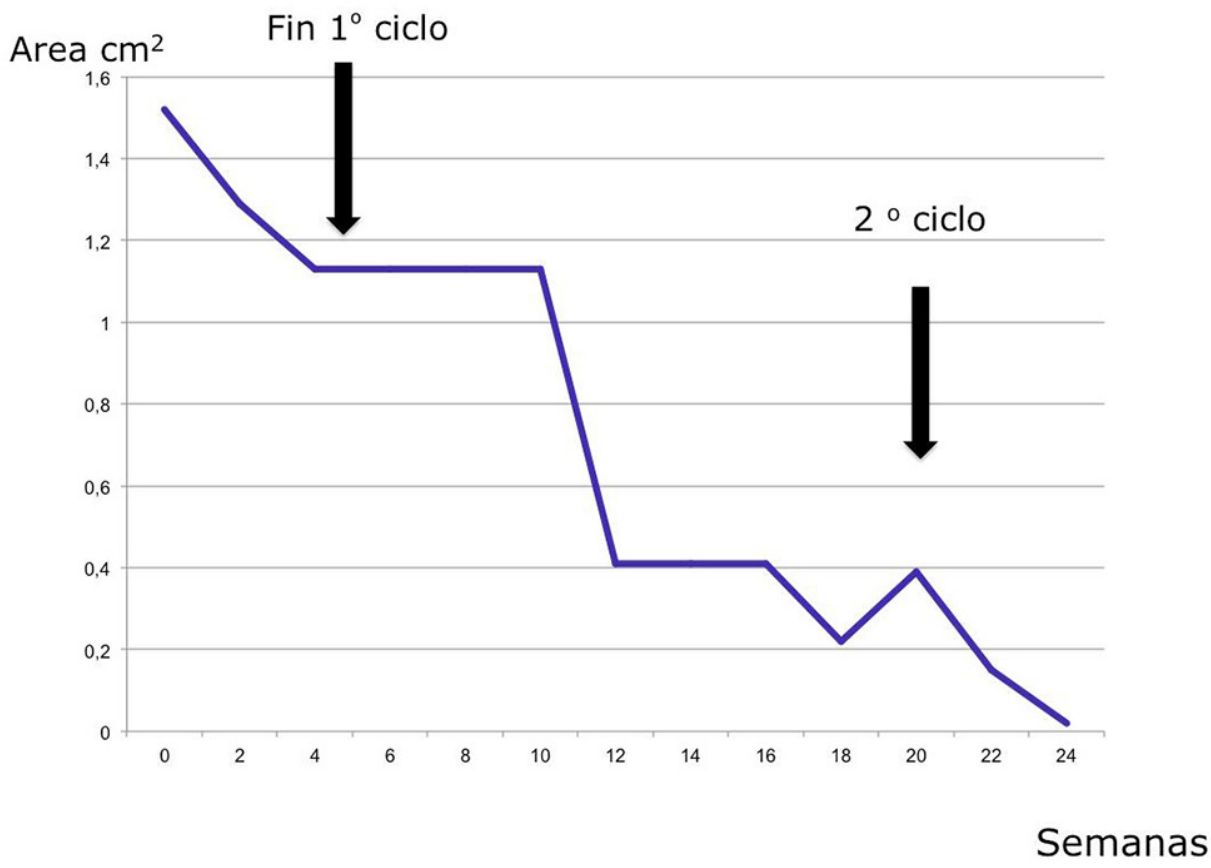

\section{Discusión}

Las heridas crónicas nos enfrentan a un desafío terapéutico sin alcanzar en ocasiones, una cicatrización óptima.

La literatura citada previamente apoya las propiedades del uso del G-CSF en lo referente a infección y cicatrización de heridas en pacientes diabéticos. ${ }^{(12-14,17)}$ Sin embargo, hasta la fecha, no hemos encontrado estudios publicados realizados in vivo en heridas crónicas de etiología venosa, que avalen la eficacia y seguridad del G-CSF en pacientes con úlceras venosas crónicas de miembros inferiores, así como tampoco en heridas crónicas en áreas de radiodermitis.

La metodología utilizada enla aplicación del G-CSF se realizó en forma similar al protocolo de Cianfarani y cols. ${ }^{(9)}$, si bien no se ha establecidoun acuerdo en cuanto al intervalo de aplicación (diaria, semanal o quincenal), así como tampoco en el número de aplicaciones a administrar, pudiendo oscilar entre tres a veintiuna dosis aplicadas diariamente.

Con respecto a la dosis, optamos por aplicar $300 \mu \mathrm{g}$ semanales intradérmicas en los dos pacientes, aunque en la literatura la misma oscila entre $5-10 \mu \mathrm{g} / \mathrm{kg}$ por inyección.

Con relación al porcentaje de cicatrización de úlcera venosa nuestros resultados fueron similares a los resultados obtenidos por Marques da Costa y Cianfarani, quienes reportan tasas globales de reducción a los tres meses de tratamiento de 27 a $73 \%$ para úlceras venosas con GM-CSF. ${ }^{(7,9)}$ El intervalo entre la primera y segunda serie en el caso clínico 1 fue más largo que lo reportado en la literatura, por motivos personales del paciente.

En heridas causadas por radioterapia se debe tener en cuenta el potencial carcinogénico de este producto. ${ }^{(20)}$ En el caso clínico 2 se descartó previamente la persistencia de patología oncológica con el informe anátomo - patológico, el cual evidenció márgenes libres de lesión. A pesar de esto, es recomendable continuar con un seguimiento clínico del paciente.

En cuanto al beneficio de G-CSF vinculado a la cicatrización, se observó el cierre completo en el caso del paciente con radiodermitis; mientras que en el paciente portador de úlcera venosa crónica, se obtuvo una reducción al finalizar las dos series de tratamiento del $37,1 \%$.

En lo referente a los efectos adversos locales nuestros resultados son similares a los mencionados por otros autores, ${ }^{(7,12)}$ destacándose en ambos casos leve dolor al momento de la inyección. En nuestros pacientes no se evidenciaron efectos adversos sistémicos, así como tampoco cambios en la paraclínica como hemograma y recuento de células CD34+.

Cianfarani y cols. reportan efectos adversos vinculados a la utilización con GM-CSF, como dolor a nivel dorsal compatible con radiculitis por lo que debió ser suspendida la aplicación del factor. ${ }^{(9)}$ 
Por otra parte, en nuestro país el costo de cada inyección de Filgen ${ }^{\circledR}$ es de 1500 pesos uruguayos $^{(21)}$, lo que es comparable al precio de algunos apósitos utilizados en curación avanzada de heridas.

Es por este motivo que consideramos que aquellas estrategias terapéuticas que logren disminuir los tiempos de cicatrización, como el G-CSF, con costos accesibles para los sistemas sanitarios, impactarán positivamente en el gasto destinado para su tratamiento.

\section{Conclusiones}

Ambos pacientes presentaron un beneficio en la cicatrización de sus heridas con la utilización de G-CSF, sin bien se requieren de más estudios para establecer el rol de este fármaco en la cicatrización de heridas.

Quedan algunas interrogantes planteadas como ser la dosis, intervalo y número total de aplicaciones a administrar, ya que no existe uniformidad en la literatura sobre este punto.

Los resultados obtenidos nos llevan a continuar con este estudio mediante el diseño de ensayos clínicos con la finalidad de validar esta herramienta terapéutica en el tratamiento de los pacientes con heridas crónicas de diversas etiologías. Para esto nuestro grupo de trabajo se encuentra desarrollando un estudio prospectivo randomizado que permita dar respuesta a algunas de estas interrogantes.

\section{Agradecimientos}

Al laboratorio Roemmers por la donación de las dosis de Filgen ${ }^{\circledR}$, apoyando de este modo la innovación en la terapia del área de medicina regenerativa.

Al Dr. Jorge Navarrete por su colaboración con el equipo de trabajo.

\section{Bibliografía}

1- Moffat CJ, Franks PJ, Doherty DC, Martin R, Blewett R, Ross F. Prevalence of leg ulceration in a London Population.QJM. 2004; 97: 431-37.

2- Ramos S, Mendez E, Labat A, Peyre A, Anda G de, Puig R, et al. Ulceras de pierna: causas infrecuentes. Rev Med Uruguay 2001;17:33-41.

3- Otero González G, Agorio Norstrom C, Martínez Asuaga M. Úlceras de miembros inferiores. Características clínico-epidemiológicas de los pacientes asistidos en la unidad de heridas crónicas de Hospital de Clínicas. Rev Med Urug. 2012; 28: 182-189.

4- Sen CK, Gordillo GM, Roy S, Kirsner R, Lambert L, Hunt TK, et al. Human skin wounds: A Major Snowballing threat to public health ann the economy. Wound Repair Regen. 2009;17 (6);763-71.

5- Alavi A, Sibbald RG, Phillips TJ, Miller OF, Margolis DJ, Marston W, et al. What's new: Management of venous leg ulcers. Approach to venous leg ulcers. J Am Acad Dermatol 2016;74:627-40.

6- Profyris C, Tziotzios C, Do Vale I. Cutaneous scarring: Pathophysiology, molecular mechanisms, and scar reduction therapeutics. Part I. The molecular basis of scar formation. J Am Acad Dermatol. 2012;66 (1):1-10.

7- Marques da Costa R, Jesus FM, Aniceto C, Mendes M. Double-blind Randomized Placebo-controlled Trial of the use of Granulocyte-Macrophage Colony-stimulating Factor in Chronic Leg Ulcers. Am J Surg. 1997;173:165-68.

8- Jaschke E, Zabernigg A, Gattringer C. Recombinant human GM-CSF applied locally in low doses enhances and prevents recurrence of chronic venous ulcers. Int J Dermatol. 1999;38:380-86.

9- Cianfarani F, Tommasi R, Failla CM, Viviano MT, Annessi G, Papi M, et al. Granulocyte/macrophage colony-stimulating factor treatment of human chronic ulcers promotes angiogenesis associated with de novo vascular endothelial growth factor transcription in the ulcer bed. Br J Dermatol.2006;154:34-41.

10- Lin S, Xu H, Xiao J, Liu Y, Zhang Y, Cai L, et al. Combined Use of Acid Fibroblast Growth Factor, Granulocyte Colony-stimulating Factor and Zinc Sulphate Accelerates Diabetic Ulcer Healing. J Health Sci. 2009; 55:910-22.

11- Eroglu E, Agalar F, Eroglu F. Effects of granulocyte-colony stimulating factor on wound healing in a mouse model of burn trauma.Tohoku J. Exp. Med.2004;204:11-6. 
Rev. urug. med. interna.

12- de Lalla F, Pellizzer G, Strazzabosco M, Martini Z, Du Jardin G, Lora L, et al. Randomized Prospective Controlled Trial of Recombinant Granulocyte Colony-Stimulating Factor as Adjunctive Therapy for Limb-Threatening Diabetic Foot Infection.Antimicrob Agents Chemother. 2001;45: 1094-8.

13- KästenbauerT,Hörnlein B, Sokol G, Irsigler K. Evaluation of granulocyte-colony stimulating factor (Filgrastim) in infected diabetic foot ulcers. Diabetologia. 2003; 46:27- 30.

14- Gough A, Clapperton M, Rolando N, Foster AV, Philpott-Howard J, Edmonds ME. Randomised placebo-controlled trial of granulocyte-colony stimulating factor in diabetic foot infection. Lancet. 1997 ;350(9081):855-9.

15- Yönem A, Cakir B, Güler S, Azal O, Corakçi A. Effects of granulocyte-colony stimulating factor in the treatment of diabetic foot infection. Diabetes Obes Metab. 2001;3:332-7.

16- Facon T, Harousseau JL, Maloisel F, Attal M, Odriozola J, Alegre A, et al. Stem Cell Factor in Combination With Filgrastim After Chemotherapy Improves Peripheral Blood Progenitor Cell Yield and Reduces Apheresis Requirements in Multiple Myeloma Patients: A Randomized, Controlled Trial. Blood.1999;94: 1218-25.

17- Cruciani M, Lipsky BA, Mengoli C, de Lalla F. Granulocyte-colony stimulating factors as adjunctive therapy for diabetic foot infections. Cochrane Database of Systematic Reviews 2013, Issue 8. Art. No.: CD006810. DOI: 10.1002/14651858.CD006810.pub3.

18- Sugiyama K, Ishii G, Ochiai A, EsumiH.Improvement of the breaking strength of wound by combined treatment with recombinant human G-CSF, recombinant human M-CSF, and a TGF-beta1 receptor kinase inhibitor in rat skin. Cancer Sci. 2008, 99:1021-28.

19- Fine JD, Manes B, Frangoul H. Systemic granulocyte colony-stimulating factor (G-CSF) enhances wound healing in dystrophic epidermolysisbullosa (DEB): Results of a pilot trial. J Am Acad Dermatol. 2015;73:56-61.

20- Haubner F,Ohmann E, Pohl F. Wound healing after radiation therapy: Review of the literature. Radiation Oncology. 2012; 7:162-171.

21- Farmanuario Uruguay. Detalle del producto: Filgen JP. Roemmers 1 jeringa prellenada de 1 ml[en línea] Montevideo: Farmanuario, 2018. [Consultado Enero 2018]. Disponible en https://farmanuario.com/ detalle-producto/?lang=uy\&id_producto=25217. 